\title{
Fluidic Control of a Turret Wake, Part I: Aerodynamic Effects
}

\author{
Bojan Vukasinovic, Ari Glezer, \\ Woodruff School of Mechanical Engineering, \\ Georgia Institute of Technology, Atlanta, GA 30332-0405 \\ Stanislav Gordeyev, Eric Jumper, \\ Department of Aerospace and Mechanical Engineering, \\ University of Notre Dame, Notre Dame, IN 46556 \\ Valdis Kibens \\ The Boeing Company, MC S306-430, \\ P.O. Box 516, Saint Louis, MO 63166
}

\begin{abstract}
The effects of direct small-scale actuation on the aerodynamic and aero-optical characteristics of the flow over a round $0.254 \mathrm{~m}$ diameter conformal optical window built into the hemispherical cap of a cylinder turret model $(D=0.61 \mathrm{~m})$ are investigated at $M=0.3$ and $R e_{D}=4.46 \cdot 10^{6}$ (with additional measurements at $M=0.4$ and 0.5 ). Flow control is effected by arrays of piezoelectrically-driven synthetic jet modules. The cumulative effect of the actuation is manifested by concomitant delay of flow separation and active, dissipative suppression of turbulent motions downstream of separation. The effects of actuation on aero-optical distortions are assessed from the flow dynamics using surface oil visualization, static pressure distributions and hot-film measurements within the separated flow domain. In addition, the suppression of optical distortions across the separated flow is estimated from Malley probe measurements over a range of elevation angles. These measurements show that for a fixed actuation level the suppression of spectral components of the optical distortion within the Malley probes's resolvable frequency band $0.5<f<25 \mathrm{kHz}$ at $M=0.3$ is about $\mathbf{3 0} \%$.
\end{abstract}

\section{Nomenclature}

$\begin{array}{ll}A_{j} & =\text { exit area of the actuator orifice } \\ A_{o} & =\text { frontal turret area } \\ C p & =\text { pressure coefficient } \\ C_{\mu} & =\text { jet momentum coefficient } \\ D & =\text { turret diameter } \\ f_{d} & =\text { actuation frequency } \\ k & =\text { turbulent kinetic energy } \\ M & =\text { Mach number } \\ \mathrm{OPD} & =\text { optical path difference } \\ \mathrm{OPD} & =\text { root-mean-square of OPD } \\ R & =\text { turret radius } \\ R e_{D} & =\text { Reynolds number } \\ S t_{D} & =\text { Strouhal number } \\ U_{0} & =\text { free stream velocity } \\ U_{j} & =\text { average jet velocity } \\ H & =\text { height of turret base } \\ \beta & =\text { azimuthal angle of pressure ports } \\ \gamma & =\text { elevation angle of optical window } \\ \gamma_{s} & =\text { flow separation angle } \\ \rho & =\text { air density }\end{array}$




\section{Background}

Control of the flow over a bluff-body turret that houses a laser-based optical system must satisfy more demanding requirements in comparison to separation control over external aerodynamic surfaces. Whereas the effectiveness of the flow control method on aerodynamic surfaces can be evaluated in terms of its effect on the time-averaged aerodynamic forces and moments, the metric becomes much more stringent when the intent of flow control is to enhance transmission of optical wave fronts through regions of separated turbulent flow. When an optical wavefront passes through a variable index-of-refraction turbulent flow near an aerodynamic surface (boundary layers, separated shear layers and wakes), its wavefront becomes distorted or aberrated and these distortions are referred as an aero-optical problem ${ }^{1}$. These wavefront distortions combined with optical aberrations caused by the wavefront propagation through the atmosphere (known as an atmospheric propagation problem ${ }^{2}$ ) ultimately degrade the light intensity from the otherwise diffraction-limited intensity at the destination. These aberrations have high spatial and temporal bandwidths which are well outside the capabilities of traditional adaptive-optic methods ${ }^{3}$. Separated shear layers are particularly destructive because of the presence of coherent vortical structures that induce strong pressure and density gradients ${ }^{4}$. Left untreated, these shear-layer-related optical aberrations can limit an airborne transmitting system to a forward-looking quadrant alone. In order to extend viewing angles to at least a portion of an aft-looking quadrant, one can extend the region of the attached flow by delaying the separation of the shear layer and/or disrupting formation of the large-scale shear layer vortices. The intent of the work presented in this paper is to evaluate the effectiveness of a strategy for achieving significant improvement in light transmission efficiency by minimizing laser wavefront degradation through active flow control suppression of the unsteady aerodynamic environment that leads to degradation of the optical environment in uncontrolled flow.

Traditional active method for controlling separation over lifting surfaces and bluff bodies makes use of the strong entrainment properties of the separated shear layer when it is excited at its unstable frequency. Introducing perturbation signals at the nominal (dimensionless) frequency $S t=\mathrm{O}[1]$ upstream of the region of separation results in enhanced entrainment and a Coanda-like deflection of the flow towards the adjacent aerodynamic surface. However, the deflected flow can separate again depending on the modified streamwise pressure gradients along the surface. While resulting in considerable changes in global aerodynamic forces, this method can degrade an optical signal that is transmitted through the affected region perhaps even beyond the levels observed in the absence of control. A body of work studying the control of separation by the application of actuation at frequencies that are at least an order of magnitude higher than the unstable frequency of the shear layer suggests that high-frequency actuation makes it possible to achieve attached flows in which large coherent vertical motions that are associated with low-frequency actuation are largely suppressed (e.g., Smith et al. ${ }^{5}$ and Amitay and Glezer ${ }^{6}$ ).

Some previous work on separated flows over a three-dimensional bluff-body configuration has been directly motivated by the aero-optical problems involving an aircraft turret. Such a configuration typically consisted of a cylindrical base having a hemispherical cap and a flat or a conformal aperture. The resulting flow field is fairly complex as shown by de Jonckheere et al. ${ }^{7}$. Investigations of flow control on these configurations included the effect of suction on the wake structure (Purhoit et al. ${ }^{8}$ ) and the addition of aft-mounted fairings and splitter plates (Snyder et al. ${ }^{9}$ ). The former showed significant alteration of the wake structure even at low levels of suction, while the latter presented reduction of the baseline drag up to $55 \%$ by using a large fairing. The separated flow behind the turret with a flat aperture and the effects of passive control on the optical aberrations were characterized by Gordeyev et al. ${ }^{10}$, while the aerodynamical and aero-optical characterization of the baseline flow field over a conformal-window turret configuration was investigated by Gordeyev et al. ${ }^{11}$. There have been few investigations of generic surface-mounted hemispheres at high Reynolds numbers. In a numerical investigation of winds over hemispherical domes ${ }^{12}$, Manhart ${ }^{13}$ found that Karman-like vortex train is shed from the hemisphere. In these flows however, the thickness of the upstream boundary layer is comparable to the hemisphere radius, unlike the flow over an aircraft turret for which the oncoming boundary layer thickness is much smaller than the turret radius. Another limiting case, when the cylindrical base is much higher than the hemisphere radius was investigated by Leder et al. ${ }^{14}$ $(H=3 R)$. They characterized the wake behind such a high aspect ratio turret and showed that it is dominated by vortices shed off the cylindrical support. The effectiveness of direct, high-frequency control $\left(S t_{D}>10\right)$ of the separated flow over a hemispherical turret on a flat plate with a thin upstream boundary layer was demonstrated by Vukasinovic et al. ${ }^{15}$ at $R e_{D}=4-7 \cdot 10^{5}$. These authors showed that the presence of flow control can substantially reduce the extent of the recirculating domain downstream of the hemisphere with significant reduction in turbulent kinetic energy. Morgan and Visbal ${ }^{16}$ performed a numerical investigation of the flow over a turret at $R e_{D}=4.36 \cdot 10^{5}$ and $M=0.5$ and compared a hybrid RANS/ILES and $k-\varepsilon$ RANS simulations with experimental data for the baseline (uncontrolled) flow. Vukasinovic and Glezer ${ }^{17}$ demonstrated the effectiveness of fluidic, direct high-frequency 
control in turbulence suppression behind a bluff-body turret at $R e_{D}=8 \cdot 10^{5}$. Recently, Vukasinovic et al. ${ }^{18}$ simultaneously measured the aerodynamic and aero-optical environment within the separated flow off a hemispherical turret at free stream Mach-number speeds up to $M=0.64$. They reported significant suppression of turbulent fluctuations and reduction in optical distortions up to $M=0.45$ by the active flow control.

The present paper reports the effects of direct small-scale excitation on the aerodynamic and aero-optical characteristics within the near wake of a cylindrical turret model with a rotatable hemispherical cap. The actuation which is effected by arrays of synthetic jet actuators results in concomitant delay of flow separation and active, dissipative suppression of turbulent motions within the separated shear layer. The effects of the actuations on aerooptical distortions are investigated over a range of optical window elevation angles and are characterized using surface pressure measurements and hot-film anemometry within the separated flow. In addition, direct aero-optical distortions are assessed using Malley probe measurements. While the primary objective of the current work is to assess the effectiveness of active flow control for suppression of optical aberrations at $M=0.3$, the control effectiveness was also assessed at $M=0.4$ and 0.5. The experimental setup and procedure are described in Section II. The characterization of the base flow is described in Section III, while Section IV presents the results of controlled flow, and finally, the conclusions are presented in Section V.

\section{Experimental Setup Procedures}

The present turret model is shown in Figure 1. It measures $0.61 \mathrm{~m}$ in diameter, and is equipped with a $0.254 \mathrm{~m}$ diameter spherical cap that models an optical window and therefore this area is unavailable for the flow control hardware. While the cylindrical turret base $(H / R=0.625)$ remains stationary, the spherical cap can be rotated about the z-axis for about $53^{\circ}$, thus allowing variation in the window's elevation angle $\gamma$ (defined as the angular position of the window's centerline, relative to the free stream). The spherical cap is instrumented with static pressure ports that are distributed along central, middle $\left(20^{\circ}\right.$ off centerline), and the outer $\left(40^{\circ}\right.$ off centerline) planes (Figure 1$)$, which include 39,44, and 39 ports, respectively. In addition to these main pressure ports, nine static pressure ports also are distributed just upstream from the optical window, such that the middle port is in the central plane, and four pressure ports are distributed over each half of the window, having azimuthal angles $\beta=12.5^{\circ}, 25.6^{\circ}, 39.7^{\circ}$, and $57.7^{\circ}$. These additional nine ports are used to assess the flow symmetry and spanwise (z-direction) effects of the actuation.

A total of 36 individually-addressable actuators are distributed in three rows around the window circumference, such that the first row (closest to the window) consists of 15, the second of 14, and the third of 7 actuators (Figure 1). Each actuator module has a high aspect ratio rectangular jet orifice (measuring $38.1 \times 0.5 \mathrm{~mm}$ ) that can be rotated about its own axis to allow different orientations of the jet orifice relative to the free stream, thus enabling predominantly streamwise or spanwise vorticity generation upon jet activation. Previous studies suggested possible advantages of the manipulation of spanwise vorticity concentrations in incompressible flows ${ }^{17}$, and of streamwise vorticity in compressible flows ${ }^{18}$. In the preliminary stages of the present investigations several combinations of orifice orientations were tested (i.e., aligned with the free stream, approximately with the local flow, and normal to the free stream), and based on these tests it was decided to utilize orifices that are approximately aligned with the free stream. Since the actuators move with the spherical turret and the optical window, their position relative to the point of separation changes and therefore different actuator arrays can be activated depending on the windows position. In the present experiments, the actuation frequency was kept at $f_{d}=1,600$ $\mathrm{Hz}$, while the actuation strength was varied over $3 \cdot 10^{-6}<C_{\mu}<$ $1.5 \cdot 10^{-5}$ per single active actuator, as the jet momentum coefficient is defined
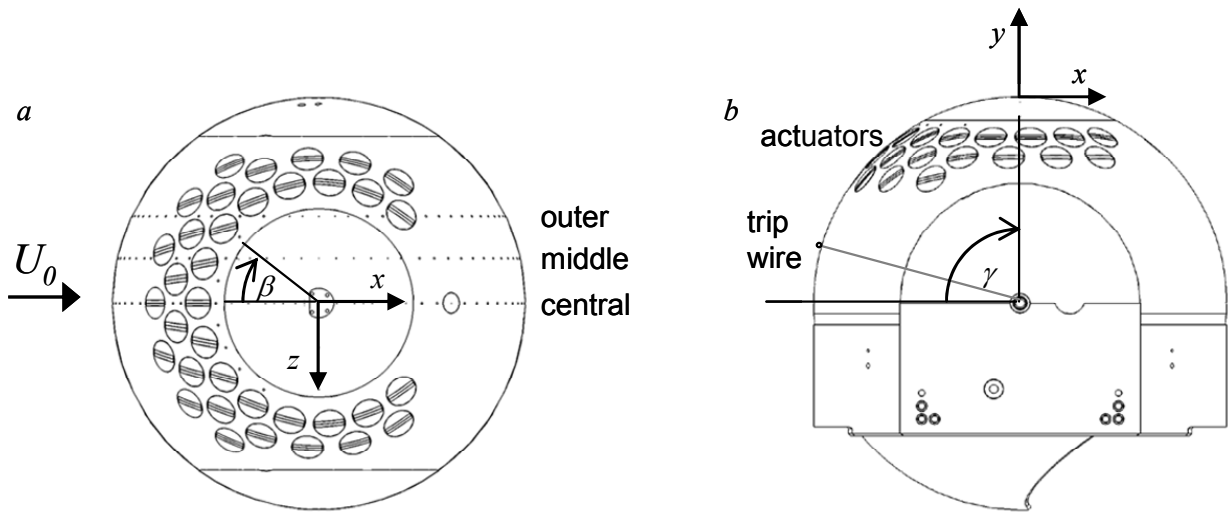

Figure 1. Top (a) and side (b) views of the $0.61 \mathrm{~m}$ dia. turret model having a $0.254 \mathrm{~m}$ instrumented spherical cap in place of an optical window. 
as $C_{\mu}=\rho U_{j}^{2} A_{j} /\left(\rho U_{0}^{2} A_{o}\right)$, where $A_{j}$ is the total jet orifice area, $A_{o}$ is frontal projection of the turret, and $U_{j}$ is the average jet velocity during the expulsion part of the cycle. Three nominal orientations of jet orifices relative to the free stream flow were tested: aligned with the free stream, normal to the free stream, and normal to the optical window center. Control effectiveness was assessed using both the full 36 actuator configuration (maximum $C_{\mu}=5.6 \cdot 10^{-4}$ ) and the center 24 actuators (maximum $C_{\mu}=3.7 \cdot 10^{-4}$ ).

Spectral characterization of the baseline (non-actuated) and actuated flows was accomplished using single-sensor hot wire anemometry. For that purpose, four hot-wire probes were mounted on retractable holders and stowed in surface wall depressions when not in use. During the measurements, only one probe at a time was elevated from the surface and positioned at the measurement location. The sensor streamwise positions are $x / R=0.5$ (HW1), 1 (HW2) 1.5 (HW3), and 2 (HW4), where $x$ is measured from the hemisphere center (Figure 1). All measurement locations are shown in Figure 2, where the cross-stream measurement locations were selected so that the local shear layer thickness was traversed.

Optical measurements were performed at the turret center plane at four window elevation angles $\gamma=129^{\circ}, 137^{\circ}, 143^{\circ}$, and $148^{\circ}$. A small surface-embedded mirror was positioned at the center of the spherical cap and Malley-probe beams were reflected off the mirror back to the optical table along the same optical path they traversed towards the turret surface. This configuration enabled a doublepass measurement through the separated flow, thus increasing the signal-to-noise ratio. Optical aberrations at these angles were measured by recording instantaneous deflection angles of both laser beams using position sensing devices. Detailed description of the optical apparatus, measurements, post-processing algorithms, and analysis of the results are presented by Gordeyev et al. ${ }^{19}$.

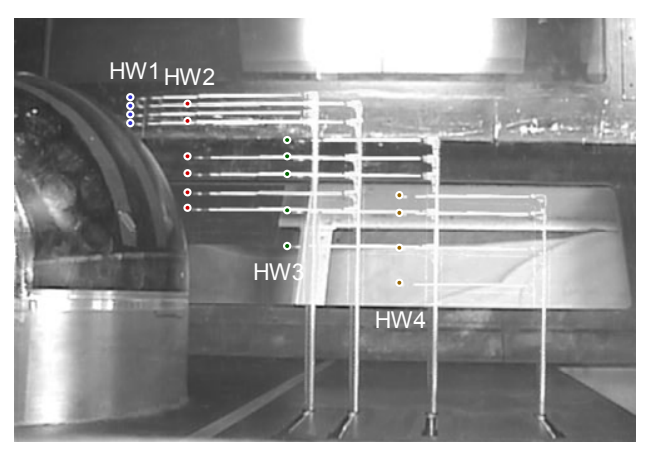

Figure 2. Overlapped measurement positions of four hot-film sensors downstream form the turret.optical window.

\section{The Baseline Flow}

Earlier investigations ${ }^{7,11,18}$ have demonstrated that the topology of the baseline turret flow is rather complex, and Mach and Reynolds number-dependent. In order to avoid changes in separation that are associated with flow transition, the hemisphere's boundary layer in the present experiments was tripped using a $0.2 \mathrm{~mm}$ dia. wire that was attached to the surface with a $0.1 \mathrm{~mm}$ tape along meridional plane at a $15^{\circ}$ elevation relative to the center (symmetry) plane of the hemisphere (Figure 1).

The tripped baseline flows are first characterized using measurements of static pressure distributions in the central, middle and outer planes shown in Figure 1. The pressure profiles in these three planes are shown in Figure 3 for window elevation angles $\gamma=129^{\circ}, 137^{\circ}, 143^{\circ}$, and $148^{\circ}$, and $M=0.3,0.4$, and 0.5 . These pressure profiles suggest that, for a given Mach number, the separation points in each of the three measurement planes nearly coincide at the planes' own reference angles, indicating that the flow separates first over the outer edges of the optical window and remains attached farthest in the central plane. This observation is in accord with previous data reported for the flow over a hemispherical turret $^{18}$. The present measurements also suggest that the separation angle in all planes shifts slightly upstream with increasing $M$, such that the flow separates just upstream from $120^{\circ}$ in the central plane at $M$ $=0.3$, while at $M=$ 0.5 , the separation point is at about $\gamma_{s}=$ $115^{\circ}$. Perhaps the
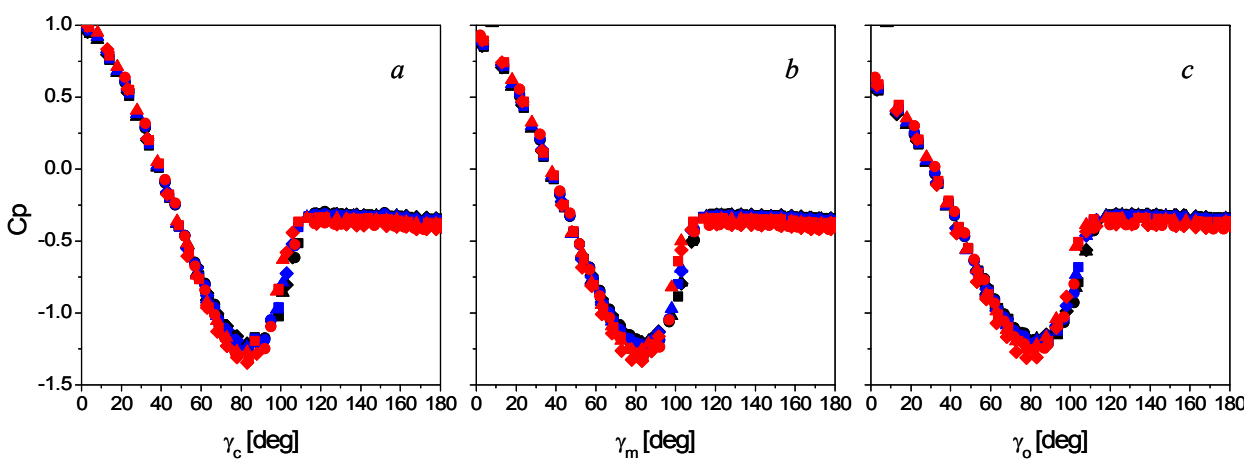

Figure 3. Overlapped static pressure measurements at window elevation angles $\gamma=$ $129^{\circ}, 137^{\circ}, 143^{\circ}$, and $148^{\circ}$, and baseline flows at $M=0.3$ (一), 0.4 (一), and 0.5 (一). in most interesting view 

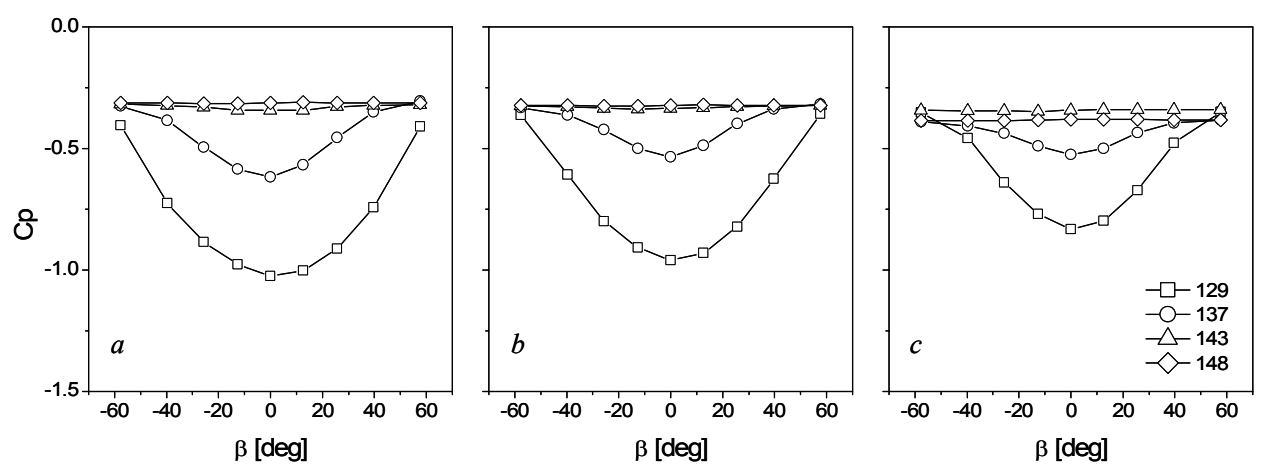

Figure 4. Pressure distributions about the optical window center $(\beta=0)$ at window elevation angle $\gamma=129^{\circ}(\square), 137^{\circ}(\mathrm{O}), 143^{\circ}(\Delta)$, and $148^{\circ}(\diamond)$, and for the baseline flows at $M=0.3(a), 0.4(b)$, and $0.5(c)$. of the baseline flow over the optical window is shown in Figure 4 which includes pressure distributions along the upstream edge of the window (cf., Figure 1) for $\gamma=129^{\circ}, 137^{\circ}$, $143^{\circ}$, and $148^{\circ}$, and $M=0.3,0.4$, and 0.5 . These data show that in all of the cases the flow remains remarkably symmetric about the streamwise centerline

of the window $\left(\beta=0^{\circ}\right)$. When the flow is not separated at the upstream edge of the window $\left(\gamma=129^{\circ}\right.$ and $\left.137^{\circ}\right)$, the pressure has a minimum at the window center, and increases towards the spanwise edges of the turret. The pressure profiles at $\gamma=137^{\circ}$ indicate a presence of a separated flow at the outer upstream edges of the optical window, and at $\gamma=143^{\circ}$ and $148^{\circ}$, regardless of $M$, the flow approaching the optical window is already separated over the measurement domain. The upstream shift in the separation line with increasing $M$ is also apparent in the pressure distributions at the lowest elevation angle, where the approaching flow is attached at $M=0.3$ and separated at $M=0.5$.

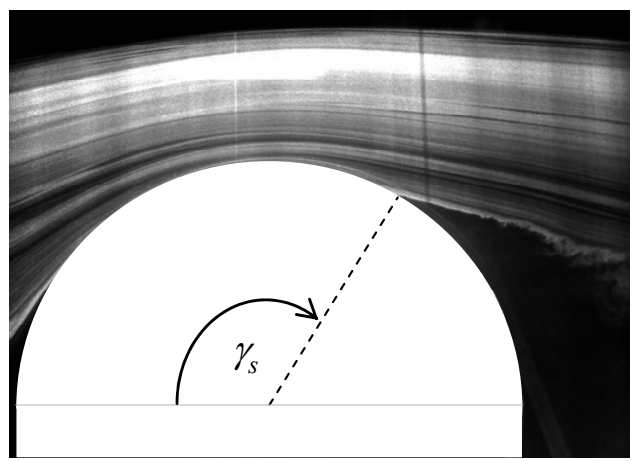

Figure 5. Visualization of the flow separation off the turret at $M=0.3$.
The baseline flow at $M=0.3$ was also characterized by flow visualization using fog particles that were injected near the tunnel wall upstream of the contraction. A snapshot of this visualization is shown in Figure 5, illustrating the separation off the turret surface in the center plane. These images indicate that the separation angle in the center plane is $\gamma_{s}=117^{\circ}$, which closely matches the corresponding static pressure profile shown in Figure $3 a$. In addition, the global topology of the baseline flow at $M=0.3$ was investigated using surface oil-flow visualization. The main footprint of the flow was visualized over the flat surface of the supporting turret wall, and additional visualization was performed along the cylindrical turret base. The recorded images of the surface oil visualization showed that the baseline flow over the turret is quite symmetric. Some features of the flow that were educed from these images are shown in schematically in Figures $6 a$ (the turret support plane) and $6 b$ (the cylinder surface). As the oncoming boundary layer approaches the adverse pressure gradient induced by the presence of the cylinder support, a spanwise (necklace) vortex is formed which becomes strained and deformed under the modified pressure field, giving a rise to the streamwise vortex branches along each spanwise edge of the cylinder. A stagnation point is formed at $0.5 R$ upstream from the turret edge, the outer flow is displaced by the bluff-body, accelerates over the hemisphere surface and around the cylindrical surface until the adverse

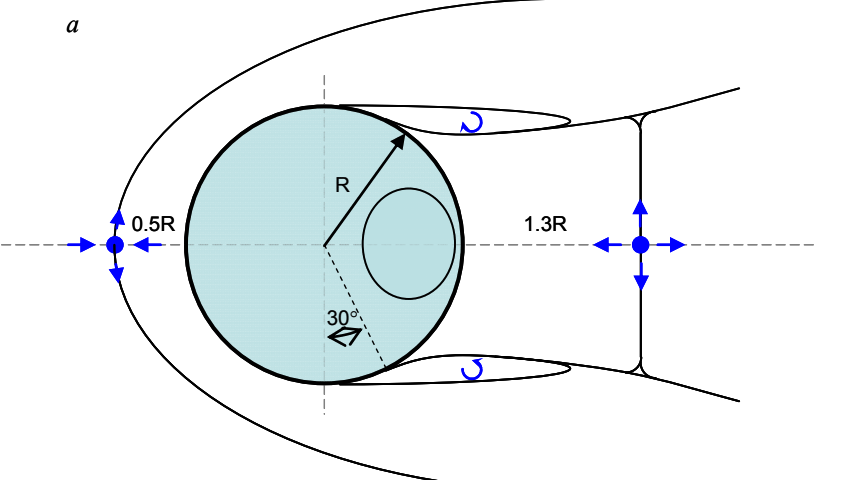

$b$

Figure 6. Schematics of the surface oil-flow visualization of the baseline flow at $M=0.3$ at the support wall (a) and cylindrical surface of the turret (b).

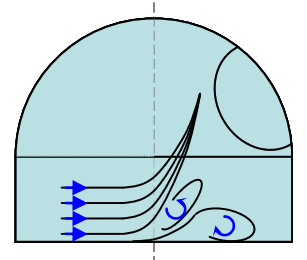


pressure gradients on the aft sides induce separation. The flow footprints shown schematically in Figure $6 a$ indicate that the flow separates off the cylinder base near its apex, and spreads azimuthally by approximately $120^{\circ}$. The visualization on the surface of the cylinder in Figure $6 b$ indicates the 3D nature of the separating flow near the cylinder's base, as well as its localized, near-wall effect. Further away from the support wall, the flow trajectories over the cylinder become displaced towards the hemispherical turret cap, and merge into a separation line. This visualization supports the assessment, based on the static pressure measurements (Figures 3 and 4), that the flow separates first at the spanwise edges of the turret and remains attached farthest in the (center) plane of symmetry. The ensuing separated flow off the turret surface has a main central wake that reattaches to the support wall at approximately $1.3 R$ downstream from the turret edge, and two additional near-wall wake segments that appear to be associated with the vortices that are shed off each side of the cylindrical support. After initial narrowing, the main wake begins to spread as shown in Figure $6 a$. It appears that the overall wake dynamics is dominated by the flow separation off the hemispherical cap, while the separation off the relatively short cylinder base has a limited effect on the wake. In contrast to this observation, measurements in a high aspect ratio turret ${ }^{14}$ where cylindrical base is much taller than its radius, the near wake is dominated by the dynamics of the vortices shed off the cylinder.

While most of the spectral characterization of the separated flow was done in connection with the evaluation of the flow control approaches, the cross-stream spreading of shear layer was monitored at the streamwise positions marked HW1 HW4 in Figure 2. An example of these data at $M=0.3$ is shown in Figure 7 which includes four spectra of velocity fluctuations measured at $x / R=0.5$ (HW1) and $y / R=-0.042$, $0.083,-0.125$, and -0.167 ( $y$ is measured from the hemisphere apex). It is seen that the two outer spectra $(y / R=-0.042$ and 0.083 ) represent the outer, high-speed edge of the shear layer and exhibit signatures of intermittent motions as is evident by the broad spectral peaks at the lower frequencies. The spectra that correspond to the lower (inner) side of the separating shear layer are nominally featureless and exhibit a broad range of

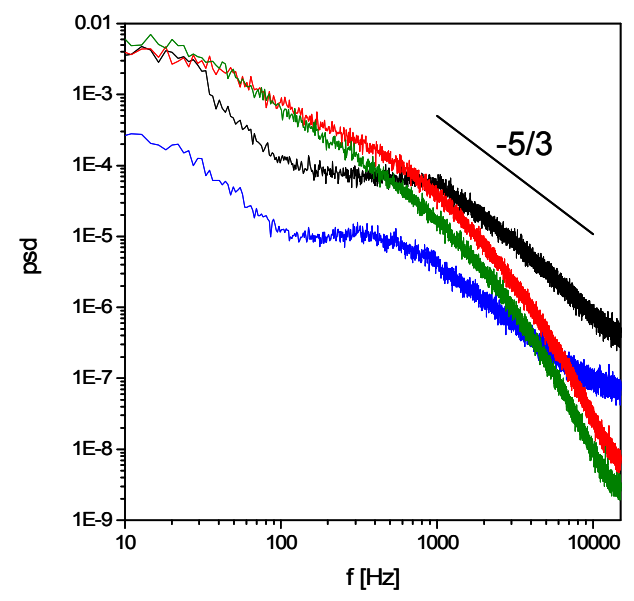

Figure 7. Power spectra of the velocity fluctuations $(M=0.3)$ measured by the HW1 at $y / R=-0.042(-),-0.083(-),-0.125(-)$, and $-0.167(-)$ turbulent motions.

\section{The Controlled Flow}

The effects of the actuation strength in terms of the synthetic jet momentum coefficient on the suppression of turbulent energy within the separated flow domain was assessed by varying the jet exit velocity $\left(C_{\mu}<5.6 \cdot 10^{-4}\right)$ and measuring the corresponding distributions of static pressure. An example of the measured pressure distributions in three planes is shown in Figure 8 for $\gamma=139^{\circ}$ and $M=0.3$. These data show a clear dependence of streamwise separation delay on the jet momentum coefficient in all three measurement planes and suggests that stronger actuation could lead to additional changes in $C_{\mathrm{p}}$. The effect of varying the jet momentum coefficient on the spectral properties of the separated flow is demonstrated in Figure 9 at a fixed elevation within the shear layer at all four hot-film measurement stations $[x / R=0.5$ (HW1), 1 (HW2) 1.5 (HW3), and 2]. As discussed
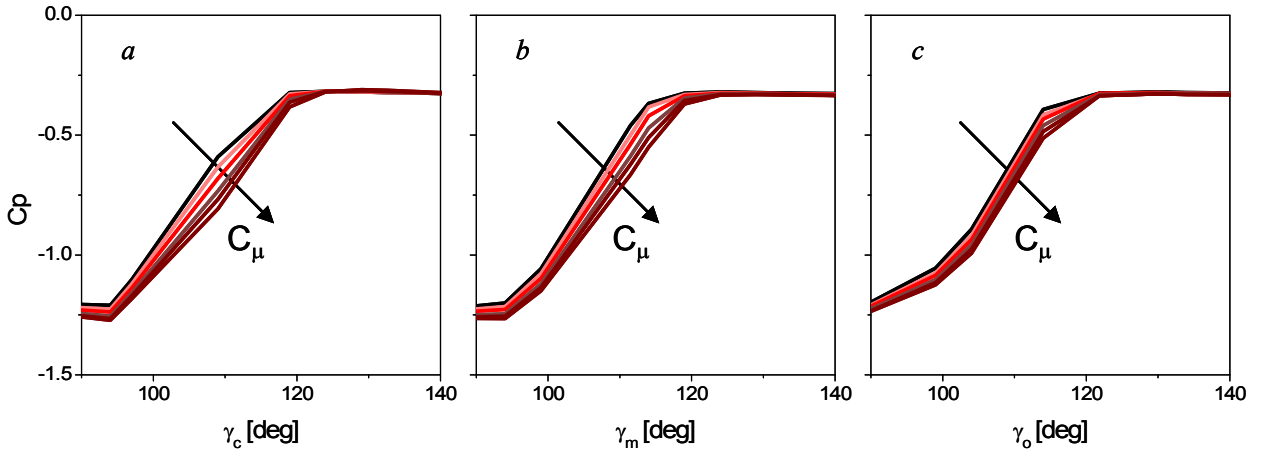

Figure 8. Static pressure profiles for the baseline flow (-) at $M=0.3$ and $\gamma=139^{\circ}$, and controlled flows in the central (a), middle (b), and the outer (c) planes. Controlled pressure profile is increasingly darker red in color with an increase in the jet momentum coefficient. 
above, direct actuation of small-scale dissipative motions leads to a significant broad band reduction in turbulent kinetic energy within the wake over the entire streamwise measurement domain. As shown in earlier investigations ${ }^{20}$, for a given jet momentum coefficient the streamwise suppression of TKE decreases with increasing distance from the control source which is a direct consequence of the enhanced dissipation within the flow. The present data also indicate that additional reduction can be attained a higher actuation power (the present measurements are taken at the maximum available actuation level $C_{\mu}=5.6 \cdot 10^{-4}$ ).

总

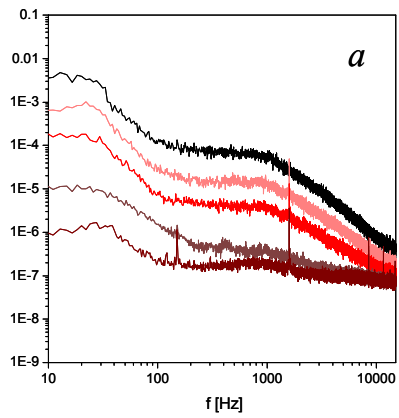

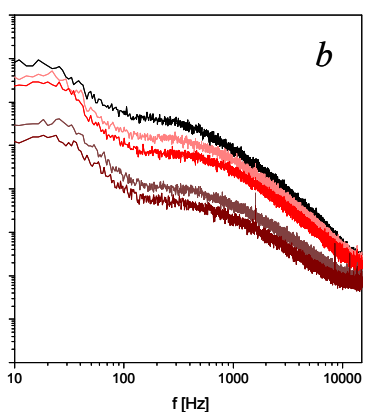
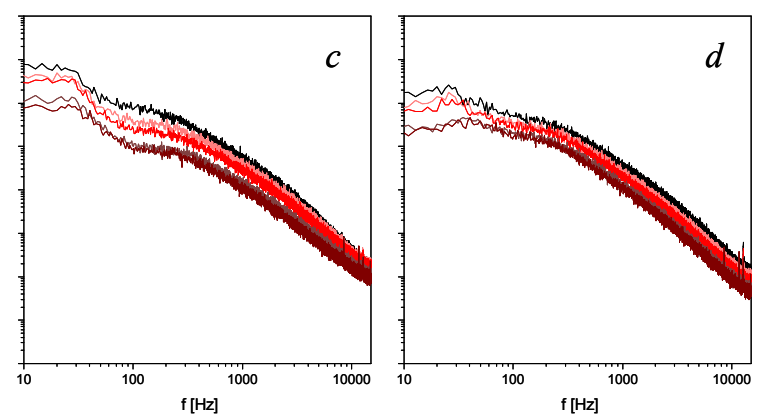

Figure 9. Power spectra of the velocity fluctuations $\left(M=0.3\right.$ and $\left.\gamma=139^{\circ}\right)$ for the baseline flow (一) and controlled flows measured by HW1 at $y / R=-0.042$ (a), HW2 at $y / R=-0.17$ (b), HW3 at $y / R=-0.42$ (c), and HW4 at $y / R=-0.58(d)$. The spectra of the controlled flow for increased jet momentum coefficient are marked by increasingly darker red traces.

Comparison of the static pressure distributions in the presence and absence of actuation shows consistent separation delay effects across the optical window, where the magnitude of the separation delay is about $10^{\circ}$ at $M=0.3$. This effect is illustrated in Figure 10, where static pressure distributions in each of the three measurement planes are shown for $\gamma=139^{\circ}$ and $M=0.3$ and 0.4. In the presence of actuation the alteration of the baseline pressure distributions begins at about $75^{\circ}$ i.e., upstream from the turret apex. The acceleration of the outer flow extends the pressure recovery in the downstream direction, which in turn delays the separation of the boundary layer. The separation delay effect becomes less pronounced with an increase in $M$ due to the effective decrease in jet momentum coefficient, as can be shown by comparison of the distributions in all three measurement planes for $M=$ 0.3 and 0.4. Although separation control typically results in some suppression of turbulent fluctuations in the separated flow, the work of Vukasinovic et al. ${ }^{20}$ has shown that dissipative actuation upstream of a separating shear layer leads to disruption of turbulent kinetic energy budget in the ensuing flow. Active suppression of turbulent kinetic energy within the shear layer can be achieved even in the absence of significant separation delay, as it was demonstrated in a shear layer separating from a backwards facing step ${ }^{20}$.

Pressure distributions just upstream of the optical window are shown in Figure 11 for the flow conditions in Figure 10. Both baseline and actuated pressure distributions indicate symmetric pressure distributions about the center
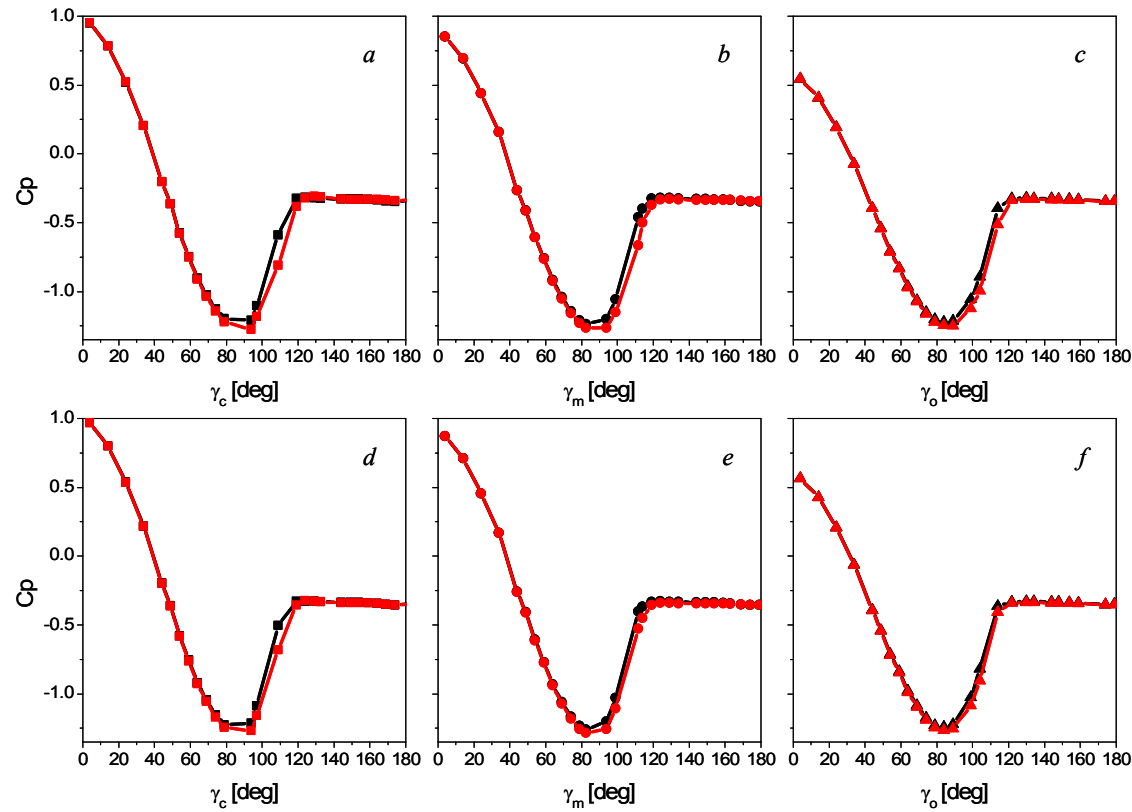
$\gamma_{\mathrm{m}}$ [deg]
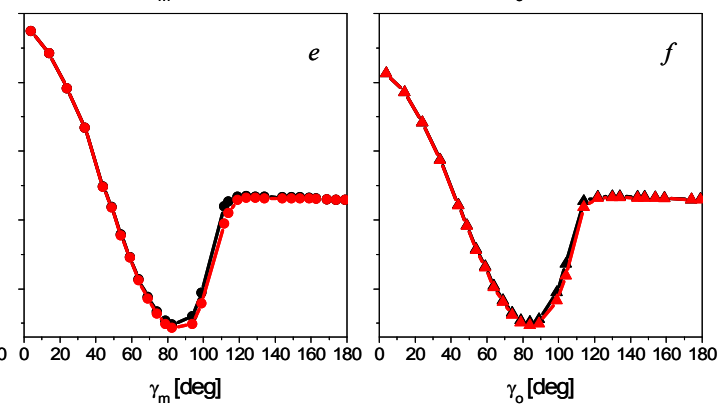

Figure 10. Static pressure distributions for the baseline flow (-) at actuated (-) flows at $\gamma=139^{\circ}$ in the central $(a, d)$, middle $(b, e)$, and the outer $(c, f)$ planes at $M=0.3(a-c)$ and $0.4(d-f)$. 

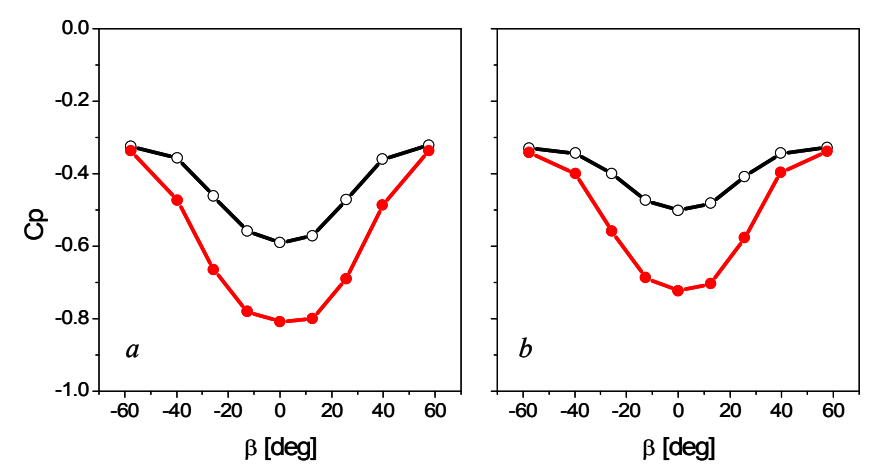

Figure 11. Pressure distributions about the optical window center $(\beta=0)$ at window elevation $\gamma=139^{\circ}$ for the baseline flow (-) at actuated (-) flows at $M=0.3$ (a) and 0.4 (b). plane of the window $(\beta=0$, Figure 1). At both Mach numbers, the baseline flow upstream of the window is attached and similar to the data shown in Figure 4 above, the inverted bell-shape profile indicates that the flow first separates at the spanwise edges of the window, while the flow over front central part of the window remains attached. In the presence of actuation at $M=0.3$ (Figure $11 a$ ), the largest reduction in pressure is achieved at the center of the window but there is a nearlyuniform separation delay over about the center half of the window span. Farther outboard towards the edges of the window $\left(\beta= \pm 57.7^{\circ}\right)$, there is a sharper decrease in the delay of separation. A similar, effect is also seen for $M=0.4$ (Figure $11 b$ ).

The differences between the baseline and controlled flows are evident from spectra of velocity fluctuations measured using hot wire anemometry (Figure 12). Overall, the spectral analysis shows significant broadband reduction in the energy of motions within the separated shear layer (it should be pointed out that at a given downstream position, the energy balance depends on the cross-stream elevation within the shear layer). The measurements at the downstream edge of the turret at $x / R=1$ (HW2 in Figure 2) were conducted at six cross-stream elevations $y / R=-0.083,-0.167,-0.25,-0.33,-0.42$, and -0.5 . Similar to Figure 9, these data show a significant broadband suppression of energy in the presence of actuation indicating a potential reduction in optical aberrations. Near the high-speed edge of the shear layer (Figures $12 a$ and $b$ ) the decrease is primarily pronounced at the low end of the spectrum indicating a substantial reduction in the advection of large-scale vertical structures. In the central segment of the shear layer (Figure 12c), there is a slight increase in energy of the small-scale motions suggesting an accelerated cascade of TKE to the small, dissipative scales. It is remarkable that near the low-speed edge of the baseline shear layer (Figures $12 d$ and $e$ ), there is a small increase in the energy of the low-frequency spectral components indicating an increase in entrainment and increased mixing at the edge of the wake. Lower within the wake, there is no significant alteration of the fluctuating energy in the presence of actuation (Figure 12f).

Further spectral analysis compares the effects among three Mach numbers tested, $M=0.3,0.4$, and 0.5 . Figure 13 shows power spectra of velocity fluctuations measured in the central zone of the baseline shear layer at all four measurement locations. It is consistent that for any effect seen at the nominal Mach number flow $M=0.3$, its analogous counterpart was also present in the higher Mach number flows, only with the weakening magnitude. For instance, closest to the control origin (Figure 13a), there is a dominant broadband suppression of fluctuation energy at $M=0.3$; similar, but weaker suppression of energy at large scales at $M=0.4$, but accompanied with an increase in energy at the small-scale motions. Ultimately, increase in energy spreads towards the lower frequencies at the highest Mach number flow, and becomes the dominant effect of the flow control. The effect at $M=0.3$ at the next downstream location (Figure 13b) is similar to the effect at $M=0.4$ at the previous location, while the
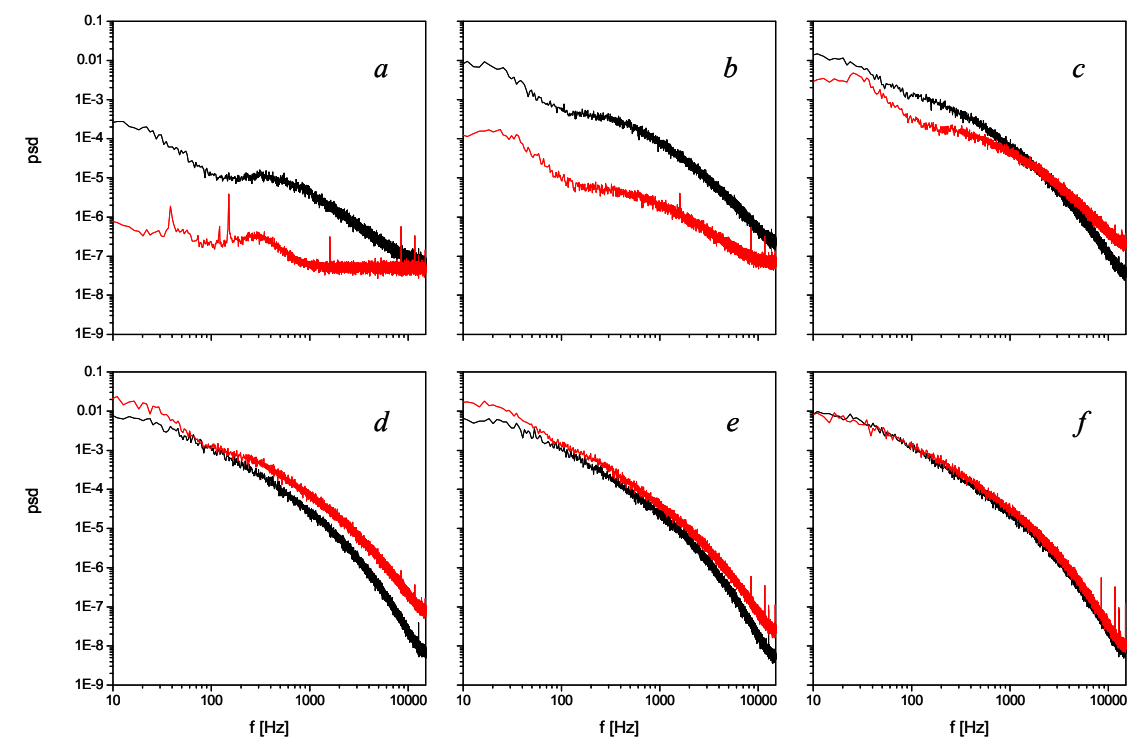

Figure 12. Power spectra of the velocity fluctuations $(M=0.3)$ for the baseline (一) and actuated flow (-), measured at $x / R=1$ (HW2) at $y / R=-0.083$ (a), $0.167(b),-0.25(c),-0.33(d),-0.42(e)$, and $-0.5(f)$. 

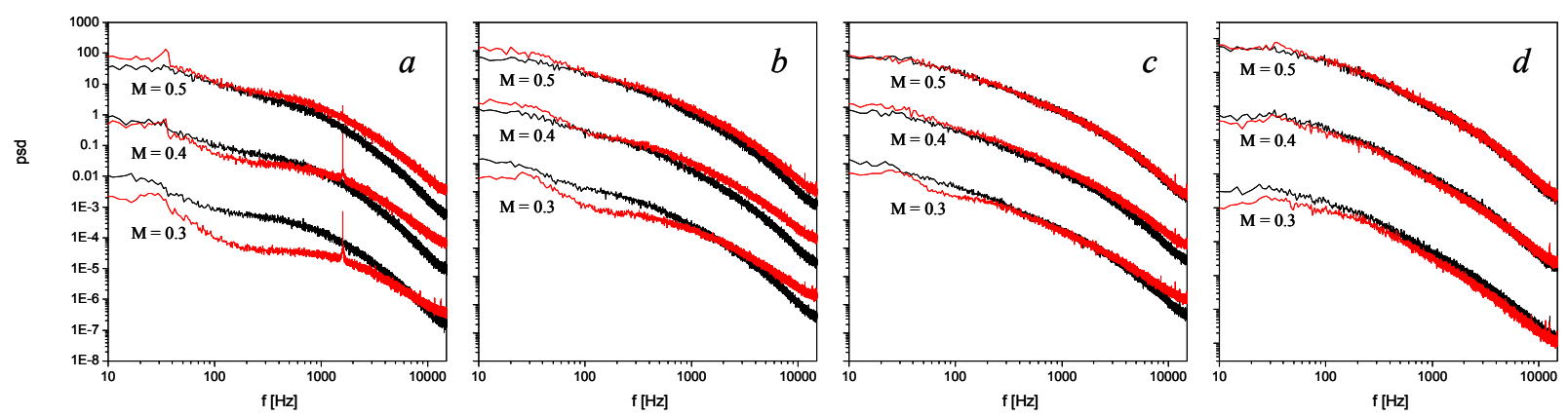

Figure 13. Power spectra of the velocity fluctuations for the baseline $(-)$ and actuated $(-)$ flows $\left(\gamma=139^{\circ}\right)$ measured by $H W 1$ at $y / R=-0.083$ (a), HW2 at $y / R=-0.25$ (b), HW3 at $y / R=-0.58$ (c), and HW4 at $y / R=-0.75$ (d) for $M=0.3,0.4$, and 0.5 .

effect at $M=0.4$ is similar to the previous location $M=0.5$, and so on. It is a consistent indicator of the weakening effect in the downstream direction (due to the dissipation of the control jets), and with the increased free-stream speed (due to decreased jet momentum coefficient). By the measurement location HW3 (Figure 13c), virtually no effect is seen at $M=0.5$, while the flows at $M=0.3$ and 0.4 exhibit some increase in energy at the small scales and some decrease of energy at the large scales. At the farthest measurement location (Figure 13d), decrease of the fluctuating energy is still present at $M=0.3$. Thus, the strongest effect of the flow control remains always closest to the control source origin, i.e., over the optical window, regardless of the free stream speed.

The effectiveness of a smaller spanwise array of actuators was investigated by comparing the effects of the center 24element segment of the 36-elemenet actuator array in Figure 1. The resulting pressure distributions upstream of the optical window are shown in Figure 14 for window elevation $\gamma=137^{\circ}$ at $M=0.3$,
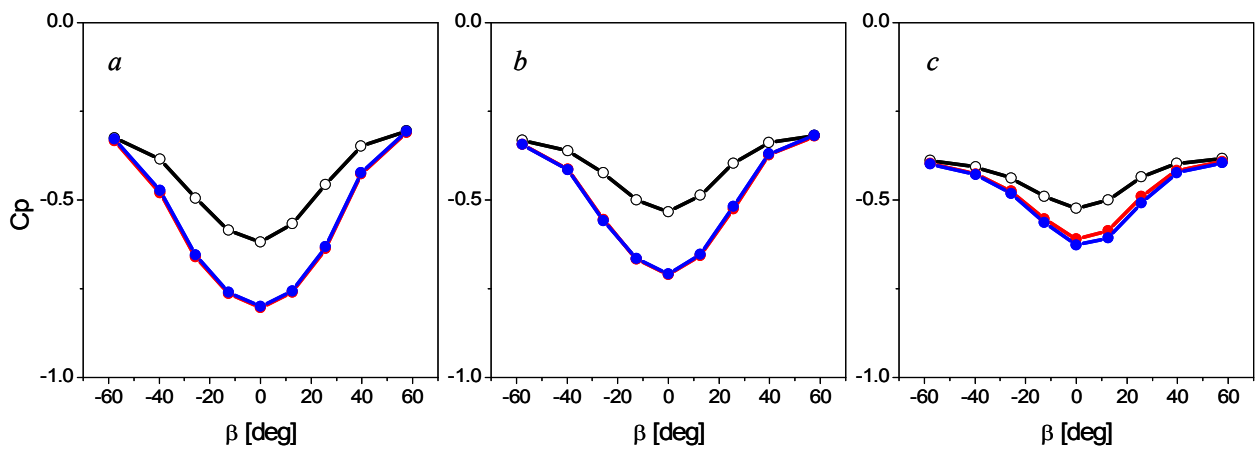

Figure 14. Pressure distributions about the optical window center $(\beta=0)$ at window elevation $\gamma=137^{\circ}$ for the baseline (-) at actuated flows by: 36(-) and 24 (一) element actuator arrays at $M=0.3(a), 0.4(b)$, and 0.5 (c).

0.4 , and 0.5 . These

data show that in all cases the effect of the smaller actuator array is virtually identical, implying that the outboard actuator segment on each side is not very effective ostensibly due to the influence of the necklace vortices that are involved in the separation of the cylinder support. It is also noted that the actuation effect becomes more localized about the window center as $M$ increases. Similarly, the effectiveness of the smaller array actuation is evaluated at three window elevations $\gamma=130^{\circ}, 142^{\circ}$, and $149^{\circ}$, as shown in Figure 15. At $\gamma=130^{\circ}$ (Figure $15 a$ ), the baseline flow is attached in front of the optical window, and the actuation delays separation uniformly across the window even with the 24-element array. At $\gamma=142^{\circ}$ (Figure 15b), the baseline flow is separated upstream of the optical window, but the actuation delays separation across the upstream edge of the window. At $\gamma=149^{\circ}$ (Figure $15 c$ ), the flow remains separated over the upstream edge of the window even in the presence of actuation (indicating that the actuation should be applied farther upstream), although, as indicated by the measured velocity spectra, the flow fluctuations within the separated domain are significantly suppressed.

The magnitude of optical distortions that would be encountered by a laser beam emerging from the optical window was measured directly using a Malley probe (Gordeyev et al. ${ }^{19}$ ). The Malley probe (MP) sensor records time series of deflection angles of two laser beams and reconstructs optical distortions using a frozen field hypothesis. Spectra of deflection angles measured in the baseline and actuated flows at $M=0.3$ are shown in Figure 16 for window elevation angles $\gamma=129^{\circ}, 136^{\circ}, 143^{\circ}$, and $148^{\circ}$. It should be noted (as is evident from these data) that accuracy of the MP measurements is in fact bandwidth-limited. The spectral optical content below $500 \mathrm{~Hz}$ is entirely masked by 

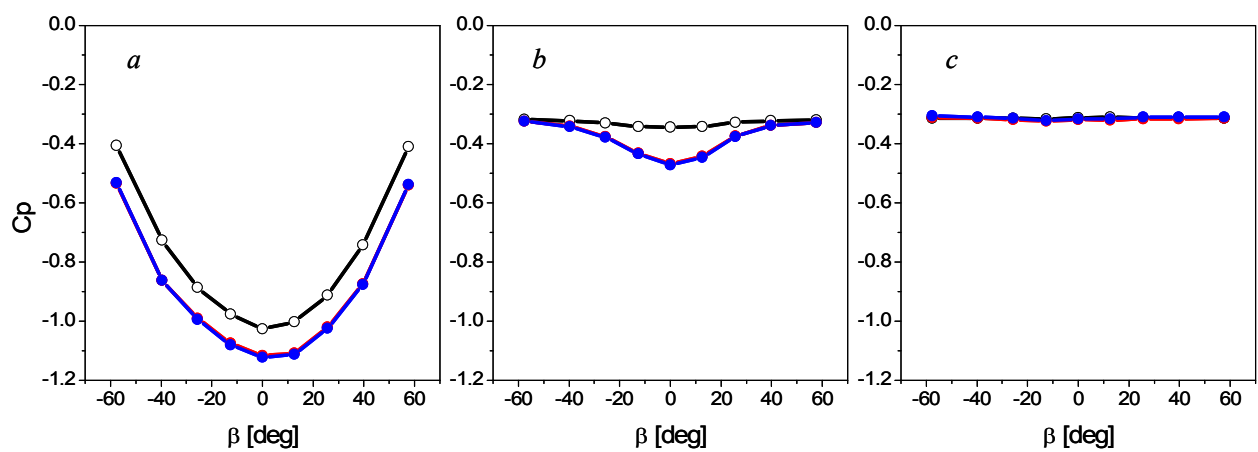

Figure 15. Pressure distributions about the optical window center $(\beta=0)$ at $M=0.3$ and window elevations $\gamma=130^{\circ}(\mathrm{a}), 142^{\circ}(\mathrm{b})$, and $149^{\circ}(\mathrm{c})$ for the baseline (-) and actuated flows using 36 (-) and 24 (-) element actuator arrays. the tunnel environment that in addition to aerooptical tunnel effects also include vibrations of the optical hardware and of the model. These vibrations which are manifested by strong spectral peaks below $500 \quad \mathrm{~Hz}$ completely overwhelm the optical signal and render the raw data unusable at these frequencies. In

addition, the present MP measurements are taken at $50 \mathrm{kHz}$, which imposes an upper frequency band limit around $25 \mathrm{kHz}$. As a result, the present MP data can only be used to assess the effectiveness of the flow control actuation within the band $500<f<25,000 \mathrm{~Hz}$. The deflection spectra of the actuated flow exhibit a peak at the actuation frequency $(1,600 \mathrm{~Hz})$ which typically dissipates rapidly as a result of enhanced dissipation. It is noteworthy that the magnitude of this peak decreases with increasing elevation angle, as the vortical motions that are induced by the actuation dissipate faster within the stronger separated base flow at the higher elevation angles. The most important effect of the actuation-induced small-scale motions on the aero-optical environment around the window is the reduction in the energy of the deflection angles within the entire frequency band that is resolved by the MP measurements at all elevation angles. In the present measurements, the reduction in spectra of deflection angles is most-significant at $\gamma=136^{\circ}$ (Figure 16b). It is interesting to note that the smallest suppression is measured at the lowest elevation angle $\gamma=129^{\circ}$ (Figure 16a), but that is attributed to a weak shear layer since the flow separates at about $\gamma_{s}=120^{\circ}$ (Figure $3 a$ ). It should also be noted that the absolute magnitude of the baseline spectrum at $\gamma=129^{\circ}$ is about an order of magnitude lower than the corresponding baseline spectrum at $\gamma=136^{\circ}$ (Figure 16b). Similar energy suppression by flow control is also measured at higher Mach-number flows, with weakening effectiveness as the actuators' momentum coefficient decreases with increasing $M^{19}$.

The measured deflection spectra were band-pass filtered within the above-stated range and the levels of optical distortions were calculated for each actuation case and compared to the corresponding baseline flow, as discussed by Gordeyev et al. ${ }^{19}$. These results are presented in Table 1, where actuation using the full set of actuators is labeled as case 1, and spanwise-limited actuation using the 24 central actuators is labeled as case 2. Full actuation (case 1) was tested at all four window elevation angles and at each Mach number, while case 2 was tested at two elevation angles for $M=0.3$ and 0.4 , and one elevation angle at $M=0.5$. As already discussed in conjunction with Figure 16, the smallest relative improvement in optical distortions within the present spectral band is measured at the lowest elevation angle at $M=0.3$, but significant suppression is measured at all other elevation
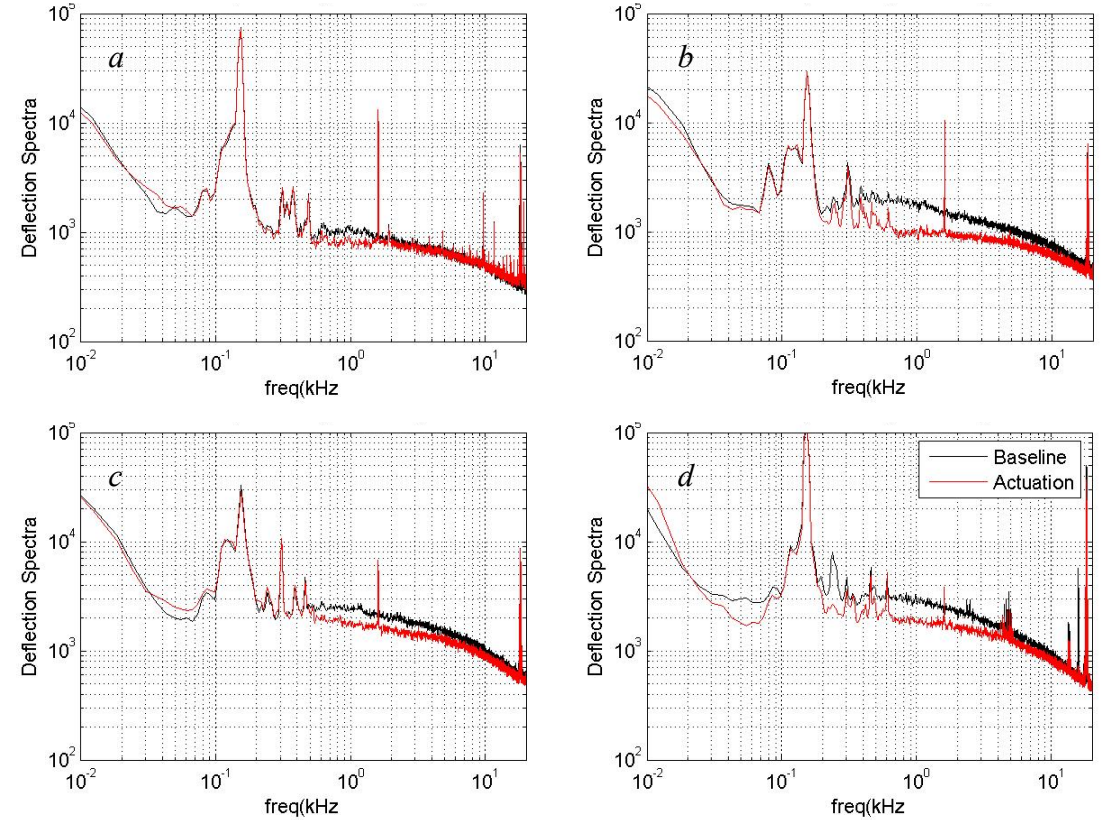

Figure 16. Spectra of the optical deflection for the baseline (-) and actuated (-) flows at $M=0.3$ and $\gamma=129^{\circ}(\mathrm{a}), 136^{\circ}(\mathrm{b}), 143^{\circ}(\mathrm{c})$, and $148^{\circ}(\mathrm{d})$. 
angles where the shear layer over optical window is more developed. Very similar, but slightly weaker effects on aberrations' suppression are achieved at $M=0.4$. The overall effect weakens further at the highest Mach-number flow $M=0.5$, but the bandwidth-limited measured suppression is still notable. When assessing the impact of reduced spatial distribution of actuators, by comparing cases 1 and 2, no significant difference is observed, as measured suppression by the spanwise-limited distribution of actuators is either slightly weaker, or even slightly stronger than in the case of full actuation. This finding suggests a possible redistribution of the control sources for future tests.

Table 1. Relative improvement in optical distortions as measured by RMS of the OPD in the actuated flows relative to the baseline flows for different elevation angles $\gamma$ based on spectral data within the range of the $M P$ sensor $0.5<f<25 \mathrm{kHz}$.

\begin{tabular}{|c|c|c|c|c|c|}
\cline { 2 - 6 } \multicolumn{1}{c|}{} & $\gamma$ [deg] & 129 & 136 & 143 & 149 \\
\hline \multirow{2}{*}{$M=0.3$} & case 1 & $8 \%$ & $34 \%$ & $24 \%$ & $28 \%$ \\
\cline { 2 - 6 } & case 2 & - & $31 \%$ & - & $37 \%$ \\
\hline \multirow{2}{*}{$\mathrm{M}=0.4$} & case 1 & $5 \%$ & $30 \%$ & $21 \%$ & $15 \%$ \\
\cline { 2 - 6 } & case 2 & - & - & $24 \%$ & $12 \%$ \\
\hline \multirow{2}{*}{$\mathrm{M}=0.5$} & case 1 & $20 \%$ & $15 \%$ & $6 \%$ & $12 \%$ \\
\cline { 2 - 6 } & case 2 & - & $12 \%$ & - & - \\
\hline
\end{tabular}

\section{Conclusions}

The effectiveness of direct, dissipative small-scale actuation for suppression of optical aberrations within the separated flow over a conformal optical window mounted in the hemispherical cap of a cylindrical turret model is investigated at $M=0.3$ and $R e_{D}=4.46 \cdot 10^{6}$. The effects of actuation on the base flow at several elevation angles of the optical window are assessed from surface oil visualization, static pressure distributions, and hot-film measurements within the separated flow domain. Suppression of optical distortions across the separated flow is assessed from direct Malley probe measurements of time series of laser beam deflection angles.

The topology of the baseline flow at $M=0.3$ indicates that the near-wake flow is dominated by the separation off the hemispherical cap of the turret. The flow separates first near the outer spanwise edges of the hemisphere, and the separation progresses towards the center plane, such that the flow remains attached farthest along the plane of symmetry. The separated wake downstream of the turret reattaches to the base plane of the turret at a nominal distance of $1.3 R$ downstream from its downstream juncture. Surface oil visualization has shown that the recirculating flow domain is bounded along its spanwise edges by two separate, narrower recirculating domains that are induced by separation off the turret's cylinder support. The flow separation along the cylinder support is amplified by interaction with the necklace vortex that forms at the juncture between the cylinder and the support plane.

Direct small-scale, dissipative actuation using arrays of streamwise rectangular synthetic jets mounted around the upstream perimeter of the optical window leads to significant separation delay on the window's surface. The extent of the separation delay varies with the elevation angle $\gamma$ of the window, and can extend up to $10^{\circ}$ at $\gamma=139^{\circ}$. Furthermore (and perhaps more importantly), spectra of the streamwise velocity fluctuations show that the dissipative actuation results in substantial broad-band suppression of the turbulent kinetic energy within the near wake and in particular the energy that is associated with large coherent vertical structures. Although this attenuation is not a direct measure of the reduction in aero-optical aberrations, it is indicative of the overall reduction in the characteristic length scales within the separated flow domain. Direct optical measurements at a free-stream Mach number $M=0.3$ show that the actuation leads to a suppression of at least $30 \%$ in optical aberration as measured within the limited frequency band $0.5<f<25 \mathrm{kHz}$ (at the low end this band is limited by tunnel optical contamination and vibrations). The broad band turbulent energy suppression that is evident from hot wire velocity spectra suggests that the actual reduction in optical aberrations may be considerably higher. Additional 
measurements at $M=0.4$ and 0.5 show similar trends albeit somewhat less effective in terms of separation delay and suppression of optical aberrations. The actuation effects at these higher Mach numbers are apparently limited by the strength of the present actuators and the precipitous decrease in jet momentum coefficient with Mach number. The suppression of optical aberrations (within the limited frequency band of the present sensor) is at least $20 \%$ and $10 \%$ for free stream Mach numbers $M=0.4,0.5$, respectively. It is noteworthy that the same suppression of optical aberrations was attained with the central $2 / 3$ subset of the jet actuator array upstream of the optical window and therefore with about $2 / 3$ of the actuation power

\section{Acknowledgment}

This work has been supported by the Air Force Research Laboratory and the Boeing Company.

\section{References}

${ }^{1}$ Gilbert, J. and Otten, L. J. (eds), Aero-Optical Phenomena, Progress in Astronautics and Aeronautics, Vol. 80, AIAA, New York, 1982.

${ }^{2}$ Tatarskii, V. I. and Zavorotnyi, V. U, "Wave propagation in random media with fluctuating turbulent parameters,", J. Opt. Soc. Am. A, Vol. 2, No. 12, 1985, pp. 2069-2076.

${ }^{3}$ Jumper, E. J., and Fitzgerald, E. J., "Recent advances in aero-optics", Progress in Aerospace Sciences, Vol. 37, 2001, pp.299339.

${ }^{4}$ Fitzgerald, E. J. and Jumper E. J., "The optical distortion mechanism in a nearly incompressible free shear layer," J. Fluid Mech., Vol. 512, 2004, pp. 153-189.

${ }^{5}$ Smith, D. R., Amitay, M., Kibens, V., Parekh, D., and Glezer, A., "Modification of lifting body aerodynamics using synthetic jet actuators", AIAA Paper 1998-0209, 1998.

${ }^{6}$ Amitay, M. and Glezer, A., "Role of actuation frequency in controlled flow reattachment over a stalled airfoil", AIAA Journal, Vol. 40, 2002, pp.209-216.

${ }^{7}$ de Jonckheere, R., Russell, J. J., and Chou, D. C., "High subsonic flowfield measurements and turbulent flow analysis around a turret proturberance", AIAA Paper 82-0057, 1982.

${ }^{8}$ Purhoit, S. C., Shang, J. S., and Hankey, W. L., "Effect of suction on the wake structure of a three-dimensional turret", AIAA Paper 83-1738, 1983.

${ }^{9}$ Snyder, C. H., Franke, M. E., and Masquelier, M. L., "Wind-tunnel tests of an aircraft turret model", J. Aircraft, Vol. 37, 2000, pp. 368-376.

${ }^{10}$ Gordeyev, S., Jumper, E. J., Ng, T. T., and Cain, A. B., "The optical environment of a cylindrical turret with a flat window and the impact of passive control devices", AIAA Paper 2005-4657, 2005.

${ }^{11}$ Gordeyev, S., Post, M. L., McLaughlin, T., Ceniceros, J., and Jumper, E. J., “Aero-optical environment around a conformalwindow turret", AIAA J., Vol. 45, 2007, pp. 1514-1524.

${ }^{12}$ Toy, N., Moss, W. D., and Savory, E., "Wind tunnel studies on a dome in turbulent boundary layers", J. Wind Eng. Ind. Aerodynamics, Vol. 11, 1983, pp. 201-212.

${ }^{13}$ Manhart, M., "Vortex shedding from a hemisphere in a turbulent boundary layer", Theoret. Comput. Fluid Dynamics, Vol. 12, 1998, pp. 1-28.

${ }^{14}$ Leder, A., Grebin, U., Hassel, E., Kashkoul, Y., and Ackl A., "3D-Flow structures behind a circular cylinder with hemispherical head geometry", Proc. Appl. Math. Mech,. Vol. 3, 2003, pp 40-43.

${ }^{15}$ Vukasinovic, B., Brzozowski, D., Glezer, A., Bower, W., and Kibens, V, "Separation control over a surface-mounted hemispherical shell", AIAA Paper 2005-4878, 2005.

${ }^{16}$ Morgan, P. E. and Visbal, M. R., "Numerical investigation of flow around a turret", AIAA Paper 2007-4480, 2007.

${ }^{17}$ Vukasinovic, B. and Glezer, A., "Control of a separating flow over a turret”, AIAA Paper 2007-4506, 2007.

${ }^{18}$ Vukasinovic, B., Glezer, A., Gordeyev, S., Jumper, E., and Kibens, V., "Active control and optical diagnostics of the flow over a hemispherical turret, AIAA Paper 2009-598, 2008.

${ }^{19}$ Gordeyev, S., Jumper, E., Vukasinovic, B., Glezer, A., and Kibens, V., "Fluidic Control of a Turret Wake, Part II: Aero-Optical Effects", AIAA Paper 2009-817, 2009.

${ }^{20}$ Vukasinovic, B., Glezer, A., and Rusak, Z., "Experimental and numerical investigation of controlled, small-scale motions in a turbulent shear layer", Proc. $3^{\text {rd }}$ International Symposium of Integrating CFD and Experiments in Aerodynamics, June 2007, U.S. AFA, CO, USA. 